\title{
El rol de la agencia de viajes ante la nueva situación post Covid-19: decálogo de propuestas para su recuperación.
}

\author{
Ricardo Pastor Ruiz* \\ Universidad de Deusto (España)
}

Jorge Rivera García**

Universitat Oberta de Catalunya (España)

\begin{abstract}
Resumen: El impacto de la pandemia del Covid-19 está siendo devastador para el sector turístico. Las consecuencias de la pandemia empiezan a verse en forma de fusiones, cierres o adquisiciones que dejarán tras de si un nuevo panorama en el subsector de las agencias de viajes. El presente estudio tiene por objeto analizar la situación de las agencias de viajes en España y, en base a este análisis, proponer un manual de buenas prácticas para su recuperación. Mediante una encuesta en línea se contactó a un total de 726 agentes de viajes. El estudio demuestra que se percibe la situación actual como un punto de inflexión a partir del cual se debe producir un cambio en el mercado que abarca desde la innovación tecnológica hasta la comercialización de productos más sostenibles, pasando por un posible cambio de modelo de negocio. Tras la observación participativa en el sector y analizar los resultados obtenidos se propone un manual de buenas prácticas articulado en forma de decálogo que, llevado a la práctica, ayudará a garantizar el futuro de las agencias de viajes ya de por si afectado ante los cambios producidos por la aparición de nuevos actores en el área de la intermediación turística.
\end{abstract}

Palabras Clave: Agencia de viajes, Intermediación; Desintermediación; Covid-19; Sostenibilidad; Omnicanalidad.

The role of the travel agency in the new post-Covid-19 situation: ten proposals for its recovery.

Abstract: The Covid-19 pandemic is having a devastating impact on the travel industry. The consequences of the virus are beginning to be seen in the form of mergers, closures or acquisitions that will leave behind a new landscape in the travel agency sub-sector. This study analyses the situation of Spanish travel agencies and, on the basis of this research, proposes a manual of good practices for their recovery. A total of 726 travel agents were contacted through an online survey. The study shows that the current situation is perceived as a turning point from which a change must take place in the market, ranging from technological innovation to the marketing of more sustainable products, including a possible change of business model. After participatory observation in the sector and analysis of the results obtained, a manual of good practices is proposed in the form of a decalogue which, when put into practice, will help to guarantee the future of travel agencies, already affected by the changes brought about by the appearance of new players in the area of tourism intermediation.

Keywords: Travel agency; Intermediation; Disintermediation; Covid-19; Sustainability; Omnichannel.

\section{Introduccion:}

La crisis de salud de COVID-19 ha tenido un impacto significativo en la actividad económica mundial, lo que ha provocado un colapso sin precedentes en el gasto de consumidores y empresas y, en consecuencia, una recesión económica mundial (Bakar y Rosbi, 2020). Las restricciones a los viajes han afectado inmediatamente a la economía mundial, incluidos los sistemas turísticos. A medida que

\footnotetext{
* Universidad de Deusto (España); E-mail: ricardo.pastor@deusto.es; https://orcid.org/0000-0002-9302-7792

** Universitat Oberta de Catalunya (España); E-mail: jriveraga@uoc.edu; https://orcid.org/0000-0003-1868-647X
} 
los viajes aéreos se han detenido o han disminuido drásticamente y muchos países han introducido períodos de cuarentena o han cerrado fronteras, el turismo nacional e internacional ha disminuido drásticamente durante un período de meses (Gössling et al., 2020).

Esto ha provocado que el turismo sea una de las industrias más afectada en esta crisis. El subsector turístico de la intermediación no ha sido una excepción, con la mayoría de empresas cerradas o en estado de hibernación durante un largo periodo de tiempo. La imposibilidad de gestionar viajes fuera de las fronteras españolas e incluso en algún momento de la pandemia dentro de las propias fronteras del país, ha hecho que la actividad comercial de las agencias de viajes haya sido muy limitada. Si a ello unimos la tendencia actual de desintermediación en la venta por parte de hoteles y compañías de transporte y la cancelación de toda la programación de los touroperadores, la actividad de las agencias de viajes desde el mes de marzo de 2020 ha sido prácticamente nula. El cierre del ejercicio 2020 para este subsector turístico ha sido pésimo. De los 20.000 millones facturados en 2019, se estima que se ha concluido 2020 con una cifra de entre 2.000 y 5.000 millones, lo que supone una caída de ventas de hasta el 90\% (Molina, 2020). La posibilidad de viajar mediante la presentación de diferentes tipos de test que aseguren la no infección del turista, no ha logrado que los ciudadanos hayan decidido viajar, teniendo en cuenta las restricciones en la movilidad dentro del propio país, de la Unión Europea y especialmente en los destinos de larga distancia. El comienzo de la vacunación masiva en los últimos días de 2020, que irá incrementándose a lo largo de 2021, se antoja como la única solución que anticipe el comienzo de una nueva etapa en el turismo mundial tras esta crisis sin precedentes desde la Segunda Guerra Mundial (Naciones Unidas, 2020).

Tradicionalmente las agencias de viajes han sido el principal canal de distribución en la intermediación turística. Durante muchos años ha sido un sector sin demasiados cambios estructurales a excepción de los que se produjeron con la irrupción de internet. Aunque muchos anticipaban el fin de las agencias de viajes tradicionales ante un previsible aumento de las transacciones directas, evitando la intermediación, la realidad ha indicado lo contrario. Incluso se ha dado la paradoja de incrementarse en algunos casos el número de intermediarios en el proceso, produciéndose una reintermediación o una hipermediación (Rodríguez-Zulaica et al., 2017). Esto hace que nos encontremos ante un nuevo escenario en la intermediación turística, que sigue evolucionando y cambiando de manera constante, y que, aunque mantiene a los actores principales, incorpora otros nuevos de marcado carácter tecnológico.

Hasta el momento, las agencias de viajes han ido adaptándose a los cambios producidos en la sociedad de la información. La información llega a los viajeros por innumerables canales creando, en ocasiones, un exceso de datos y una intoxicación informativa que hace necesario depurarla, contrastarla y sintetizarla para que el cliente pueda asimilarla. De hecho, a las clásicas funciones de asesoramiento, mediación y producción tradicionales de las agencias de viajes, se ha sumado una nueva función de igual o mayor importancia como es la de la información (Rodríguez et al., 2017). El valor añadido que ofrecen los nuevos intermediarios es el de filtrar la gran cantidad de información que el cliente recibe desde diferentes medios. Por lo tanto, en el escenario actual, el acceso y la forma de trabajar la información se puede considerar como la principal característica y el principal desafío con el que se encuentran los agentes, tanto los tradicionales, como los nuevos agentes incorporados a la cadena actual de intermediación.

La pandemia ha afectado a todo el planeta y son muchos los ciudadanos que han visto en esta crisis la posibilidad de realizar un cambio, o al menos, realizar cambios en su forma de vida, de relacionarse y por supuesto de viajar. Las agencias de viajes deben ser proactivas en este aspecto y tratar de cambiar con la sociedad. En estas nuevas circunstancias no es sorprendente que algunos autores (Cheer, 2020; Gössling et al., 2020; Higgins-Desbiolles, 2020; Jiang \& Wen, 2020; Kunzmann, 2020) argumenten que la crisis actual puede ofrecer una oportunidad para repensar y reorientar el turismo creando productos más sostenibles y de esta manera lograr un turismo más responsable.

Ante el escenario de una cercana reactivación del turismo mundial, y por lo tanto de los componentes de la tradicional cadena de valor de la intermediación (Pastor, 2019) en este trabajo se sugieren diferentes propuestas para lograr el reposicionamiento de las agencias de viajes en el mercado tras la pandemia del Covid-19, para poder llevar a cabo una reapertura del sector con una mayor garantía de éxito. Estas propuestas surgen a raíz de la observación participativa y un análisis metodológico cuantitativo, con el fin de obtener los datos estadísticos necesarios para concluir que el futuro de las agencias de viajes pasa por la aplicación más o menos rigurosa del decálogo presentado. 


\section{Marco teórico}

\subsection{Pandemia de la COVID-19}

En diciembre de 2019, Wuhan, capital de la provincia de Hubei en China, se convirtió en el centro de un brote de neumonía de causa desconocida, que atrajo una gran atención no solo dentro del país asiático sino a nivel internacional (Huang et al., 2020). De este brote, las autoridades chinas informaron por primera vez a la Oficina local de la Organización Mundial de la Salud (OMS) el 31 de diciembre de 2019. El brote se declaró emergencia de salud pública de interés internacional el 30 de enero de 2020 y el 11 de febrero de 2020 la OMS puso nombre a esta nueva enfermedad causada por un coronavirus: COVID-19 (OMS, 2020). El 11 de marzo de 2020, la OMS declaró el nuevo brote de coronavirus como pandemia mundial.

Agencias de Viajes

No es la primera vez que a lo largo de los años las agencias de viajes han tenido que enfrentarse a cambios de modelo y variaciones en el mercado. Tras la irrupción de internet, fueron muchos los expertos que vaticinaron una progresiva desaparición de las agencias de viajes (Buhalis y Licata, 2002) introduciendo conceptos relacionados con la desintermediación (Doherty et al., 1999). Otros autores contemporáneos defendían que el comercio electrónico reduciría la influencia de los intermediarios durante la cadena de valor de la experiencia turística. Esto no ha sido así. En todo caso los intermediarios han evolucionado y han utilizado la red para actualizar y adaptar su negocio, viendo en internet una ayuda y un aliado y no una barrera o un enemigo siendo hoy más fuertes que hace 20 años (Alberto et al., 2011). En un primer momento, la evolución de la tecnología provocó el desarrollo de las agencias de viajes en línea (OTA) que han sustituido en muchos casos a las agencias de viajes tradicionales (Coyle y Yeung, 2016). Debido a esto, se ha modificado considerablemente la cadena de valor de la distribución turística difuminando sensiblemente los límites entre los consumidores y los proveedores de servicios turísticos. De esta manera, clientes, productores e intermediarios crean relaciones comerciales entre sí que antes resultaban imposibles. Actualmente, es la innovación y la tecnología lo que aporta un valor añadido que deben aprovechar las agencias de viajes (Gomis y de Borja, 2006). Estos autores afirman que se ha evolucionado de la intermediación (en la que se ponía en contacto a la oferta y la demanda) a la infomediación (en la que la filtración y la selección de la información es lo que representa el valor añadido del intermediario) para llegar a la innomediación (en la que la incorporación de procesos de innovación en las empresas intermediarias supone la mayor diferencia entre los intermediarios). Y de esta manera, la transformación de la distribución y comercialización turística, ha dado lugar a un nuevo mapa multicanal en el que se desarrollan nuevas estrategias y aparecen nuevos actores.

En esta nueva era del turismo post Covid se considerarán esenciales variables como la masificación del destino, el tamaño del alojamiento, el volumen de huéspedes del mismo (incluso unifamiliares), las certificaciones Covid Free actividades con aforos limitados, al aire libre y con el distanciamiento social necesario; todo en aras de la tan ansiada garantía sanitaria. (Rivera y Pastor, 2020).

\section{Metodologia}

El objetivo de la investigación es comprobar y ratificar diferentes actuaciones que en los últimos años se están realizando en las agencias de viajes para finalmente ofrecer un manual de buenas prácticas con posibles puntos de mejora en su recuperación tras el fin de la pandemia. Se ha utilizado una metodología cuantitativa, con el fin de obtener datos estadísticos para el análisis de las hipótesis planteadas. La obtención de datos se ha realizado mediante una encuesta online durante el periodo de confinamiento más severo de 2020 con un cuestionario normalizado a una muestra no aleatoria seleccionada por conveniencia. Se ha optado por la técnica de la encuesta estructurada utilizando un formulario de Google al que se ha invitado a participar a través de redes sociales, envío de emails a agentes y a colectivos (grupos de gestión de agencias de viajes y redes turísticas). De acuerdo con los resultados obtenidos se puede señalar que el perfil de los agentes de viajes que han participado en la encuesta, en función de su edad y del tipo de agencia de viajes en la que desarrollan su actividad, es el que se señala a continuación en la tabla 1 . Se puede constatar que la gran mayoría (80,9\%) se sitúan en las franjas de edad de 31 a 55 años y casi la mitad (48,9\%) trabaja en agencias de tipo minorista dedicadas al turismo vacacional. 
Tabla 1: Perfil del encuestado

\begin{tabular}{|c|c|c|c|c|c|}
\hline Edad & $\mathbf{1 8 - 3 0}$ años & $\mathbf{3 1 - 4 5}$ años & $\mathbf{4 6 - 5 5}$ años & $\mathbf{5 6 - 6 5}$ años & $+\mathbf{6 6}$ años \\
\hline & $4,30 \%$ & $41,40 \%$ & $39,50 \%$ & $14 \%$ & $0,30 \%$ \\
\hline $\begin{array}{c}\text { Tipo de } \\
\text { agencia }\end{array}$ & Mayorista & $\begin{array}{c}\text { Minorista } \\
\text { vacacional }\end{array}$ & Receptivo & $\begin{array}{c}\text { Corporativo/ } \\
\text { empresas }\end{array}$ & Otros \\
\hline & $28,20 \%$ & $48,90 \%$ & $4,30 \%$ & $7,50 \%$ & $11,10 \%$ \\
\hline
\end{tabular}

Fuente: Elaboración propia

La muestra de la investigación la forman un total de 726 registros. Dicha población objetivo se considera bien delimitada y definida en sus características bajo el criterio de trabajar todos en la actividad turística de las AAVV de España que permite que la investigación se pueda llevar a cabo bajo condiciones correctas y fiables. La media anual entre la cifra de trabajadores afiliados a la Seguridad Social en el sector fluctuó entre los 61.384 del mes de enero y los 68.641 del mes de junio. Consideramos una media anual entre ambas cifras que arroja un universo de 65.012 agentes. De esta manera, partiendo de una muestra de 726 registros obtenidos, el error muestral se sitúa en el 3,62\%, con un índice de confianza del 95\%.

Los datos primarios generados se han analizado mediante estadística descriptiva. Los resultados se obtuvieron mediante el análisis estadístico de los datos recopilados, utilizando el programa de software libre R.

En consecuencia, el trabajo se ha basado en los datos empíricos, así como en el estudio bibliográfico y en la observación participativa en el sector de ambos autores.

\section{Resultados y discusión}

Las agencias de viajes se enfrentan a un futuro distinto, y no sólo ante el previsible cambio de escenario en cuanto a las empresas participantes, sino ante los propios clientes. Es probable que algunos vean en ellas la mejor forma de lograr un viaje seguro y sin sobresaltos y otros optarán con mayor convicción por la compra directa de servicios turísticos. En esta situación las agencias de viajes deberán adaptarse de nuevo a otro cambio importante a raíz de la mayor crisis que ha sufrido el sector en toda su historia.

Tras la revisión bibliográfica y el análisis de los resultados de la encuesta realizada entre agentes de viajes, se propone un manual de buenas prácticas idóneo para el nuevo escenario que se presenta tras la pandemia causada por la Covid-19. Se resumen en el siguiente decálogo, consejos para una reapertura organizada y rentable de las agencias que garantice el reposicionamiento de las mismas en el mercado tras la crisis.

\subsection{Desarrollo tecnológico}

Hace dos décadas, coincidiendo con la irrupción de internet en el sector de la intermediación turística, autores de referencia como D. Buhalis anunciaban que la clave del éxito futuro en la industria del turismo competitivo estaría determinada por una combinación de TI (Tecnología de la Información), intelecto y visión de gestión. La industria del turismo vive en un mundo que cambia rápidamente y necesita adaptarse constantemente para poder satisfacer las necesidades de la demanda y al mismo tiempo seguir siendo competitivo (Buhalis, 2000). Actualmente se puede comprobar que es así, e incluso a corto plazo, el desarrollo tecnológico será más importante si cabe.

Se implementarán algunas herramientas que el confinamiento y el tele trabajo han convertido en imprescindibles, como la firma y el certificado electrónico o las reuniones vía webcam, ya sean éstas con clientes, proveedores o entre compañeros de trabajo.

La Realidad Virtual (RV) será junto a la inteligencia artificial, el big data y el uso de robots, una de las tecnologías de la información que más importancia tendrán en la industria turística (Bowen y Whalen, 2017). "La RV es una técnica de visualización que superpone información digitalizada con el objeto de incorporar a la persona en un entorno que mejora su capacidad cognitiva y hace que lo aprecie como si fuera real" (Alonso Almeida, 2019). 
Actualmente se está de acuerdo en que venden más las emociones que la racionalidad. Este concepto es el que se conoce como Economía de la Experiencia. Autores como Petrus (2004) anunciaba que las empresas ya sólo venden experiencias y para que las campañas surjan efecto se deben cumplir dos premisas: 1) Involucrar al cliente en la construcción de la experiencia y 2) que todas las personas, procesos y operaciones de la organización contribuyan al desarrollo de la experiencia. La Realidad Virtual puede convertirse en un gran aliado de la industria turística y específicamente de las agencias de viajes ya que puede hacer partícipe al cliente al experimentar el viaje desde un primer momento hasta poder construirse su propia experiencia (Alonso, 2019). Actualmente esta tecnología está siendo infrautilizada por las agencias de viajes y puede ser un arma de venta muy importante si tanto destinos como proveedores desarrollan el software necesario para su utilización

El posicionamiento SEO (Search Engine Optimization, optimización para motores de búsqueda) será muy importante en el uso canal de la venta online. Trata del posicionamiento natural de una web, es decir, la facilidad con que los buscadores encuentran y reconocen una determinada web como un sitio de confianza. Cuanto mejor sea el posicionamiento mayor será la posibilidad de que esa web sea visitada por un usuario que esté realizando una consulta concreta y se produzca una conversión (o venta). Generalmente el posicionamiento SEO va acompañado de acciones de marketing como la búsqueda de enlaces relevantes y la participación en redes sociales, facilitando así la propagación y presencia en internet. La aplicación de técnicas SEO aporta las siguientes ventajas según (Ruiz y Bastidas, 2017):

- Aumento del número de visitantes que están buscando un servicio propio o producto.

- Mayor número de conversiones.

- Reputación online. Incremento de ganancias.

- Ahorro económico en campañas de marketing y publicidad tradicional.

- Carga de la página web más rápida y eficiente.

- Usabilidad

La visibilidad en redes y medios sociales es ya un hecho. La utilización sistemática de smartphones (teléfonos inteligentes) hace que la presencia en redes sociales sea obligada para poder competir. La inmediatez de lo que se sube a las redes hace que, en función de los seguidores, esa información llegue a miles de personas al mismo tiempo. Es muy importante tener visibilidad, ser ágil a la hora de contestar posibles comentarios, ya sean buenos o malos y analizar las reacciones del público al que se dirigen para poder actuar en función de lo que se genere.

Será muy importante el desarrollo de la inteligencia artificial con programas de predicción de productos y destinos en base a las necesidades y demandas de los clientes. La inteligencia artificial consiste en interpretar el mundo como los humanos lo hacen, aprendiendo a medida que actúan. Mediante el uso de algoritmos matemáticos, patrones de datos y otra información, pueden aprender relaciones complejas y tomar decisiones (Jones et al., 2018) de manera que el propio ordenador vaya autocorrigiendo los errores y mejorando sus capacidades. Actualmente esta tecnología la utilizan grandes OTA (Online Travel Agency) ya que resulta demasiado costosa, pero a corto plazo su utilización será muy importante para la mejora de la eficiencia en los procesos de venta.

\subsection{Eficiencia en los procesos}

Las organizaciones empresariales, y las empresas turísticas más si cabe, deben orientar los esfuerzos hacia la adecuación al complejo escenario en el que se mueven. Constantes cambios en las reglas de juego, incremento de la competencia o un desarrollo tecnológico muy rápido, hacen que el cliente sea más exigente y varíen constantemente sus demandas y necesidades. En los últimos tiempos, se observa también un desplazamiento del centro de interés de los sectores comerciales de las empresas, desde la promoción, al marketing de atención al cliente. En esta línea, el concepto de calidad, por ejemplo, ha pasado de ser una propiedad inherente al producto o servicio, a resultar un valor asociado a la satisfacción de necesidades y expectativas del cliente (Mallar, 2010).

El centro de todos los procesos es el cliente, por lo que, de la mano de herramientas tecnológicas adecuadas, se deben focalizar las fuerzas en ellos. Se deben eliminar actividades que no aportan valor para centrarse en la consecución de la excelencia detectando oportunidades de mejora y desarrollándolas. Todas las acciones realizadas en la empresa deberán de tener un rigor y un sentido claros, previamente estudiados y de esta manera evitar pérdidas de tiempo y por consiguiente de dinero. 


\subsection{Focalización en productos sostenibles}

La demanda de productos sostenibles es un hecho y se va a ver multiplicada en los próximos años. La necesidad por parte de los clientes de sentirse cómodos en entornos seguros, no masificados y en el que la comunidad local se sienta partícipe de la experiencia del viajero será fundamental. Se debe instar a los productores de viajes, ya sean Tour Operadores, DMOs (Destination Marketing Organization) o DMCs (Destination Management Company) a la creación de productos sostenibles para poder ofertar a los clientes. En todo caso no sólo hay que esperarlo, sino que la agencia de viajes actual debe buscarlos y proponerlos para poder operarlos. El turismo será sostenible o no será (Hall, 2019).

En el trabajo de campo realizado se puede observar en la tabla 1, que un 24,24\% de los agentes encuestados consideran que ya existía un cliente comprometido y sensible con la sostenibilidad turística. Es de suponer que tras la pandemia el número de clientes preocupados por este tema será mucho mayor.

Tabla 2: Existencia actual de cliente con perfil responsable en AAVV

\begin{tabular}{|c|c|c|c|c|c|c|}
\hline & & \multicolumn{5}{|c|}{$n=726$} \\
\hline & & $\begin{array}{c}\text { Totalmente } \\
\text { en } \\
\text { desacuerdo }\end{array}$ & $\begin{array}{l}\text { Bastante en } \\
\text { desacuerdo }\end{array}$ & $\begin{array}{c}\text { Ni de } \\
\text { acuerdo } \\
\text { ni en } \\
\text { desacuerdo }\end{array}$ & $\begin{array}{c}\text { Bastante } \\
\text { de acuerdo }\end{array}$ & $\begin{array}{l}\text { Absolutamente } \\
\text { de acuerdo }\end{array}$ \\
\hline \multirow{2}{*}{$\begin{array}{l}\text { Existencia cliente } \\
\text { interesado en } \\
\text { turismo sostenible. }\end{array}$} & $\mathrm{n}$ & 112 & 208 & 230 & 92 & 84 \\
\hline & $\%$ & $15,43 \%$ & $28,65 \%$ & $31,68 \%$ & $12,67 \%$ & $11,57 \%$ \\
\hline
\end{tabular}

Fuente: Elaboración propia

En el gráfico 1 se pueden ver cuáles son las variables o criterios del viaje sostenible que más peso han ganado en las decisiones de compra hasta el momento actual. Con diferencia, lo más valorado es un tipo de viaje menos masificado (75\%), seguido por el uso de hoteles ecológicos $(23,6 \%)$ y por el uso prioritario de productos locales o de cercanía entre los proveedores. Todavía se está lejos de que el cliente se plantee más seriamente la compensación de la huella de carbono en sus desplazamientos $(4,4 \%)$ o que se interese por los transportes menos contaminantes $(7,1 \%)$.

\section{Gráfico 1: Variables viaje sostenible con mayor peso en decisión de compra}

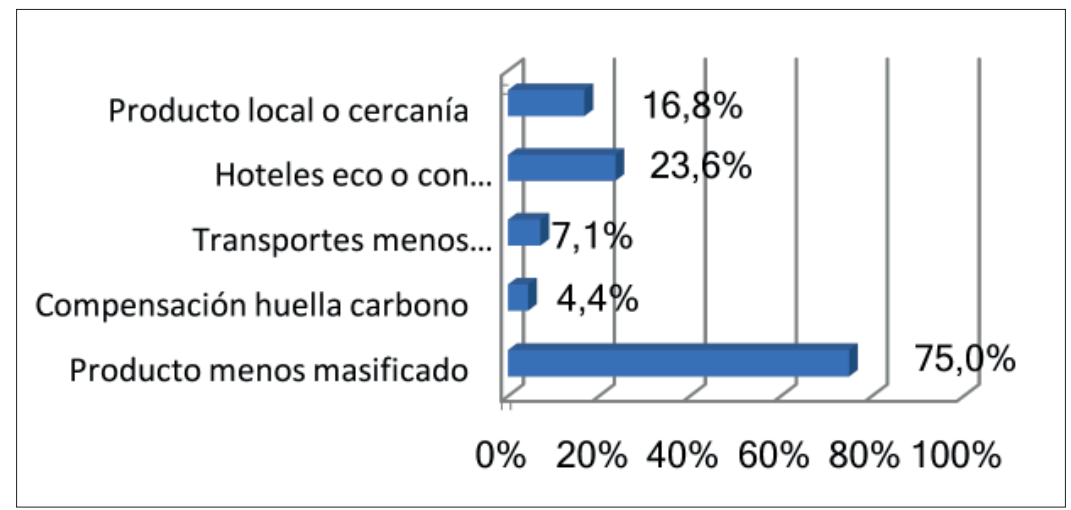

Fuente: Elaboración propia

Con relación a la percepción futura del agente de viajes sobre el nicho de mercado de turismo sostenible en la era post-COVID-19, se puede ver como casi la mitad de la muestra $(43,8 \%)$ cree que después de pandemia crecerá el cliente responsable y comprometido con la sostenibilidad en detrimento del turista tradicional ya que los principios del turismo sostenible se relacionan con una percepción más cercana 
al Covid-free siendo sólo un 20,6\% los contrarios a esta percepción. Para una gran mayoría (cerca del $78 \%$ ) de los agentes encuestados se debería aprovechar esta situación para tratar de afianzar este tipo de producto en el mercado, aunque esto choca con la realidad actual en la que casi dos terceras partes $(63,36 \%)$ consideran que el factor precio va a seguir siendo determinante en la decisión de compra.

Tabla 3: Percepción sobre turismo sostenible en era post-COVID-19

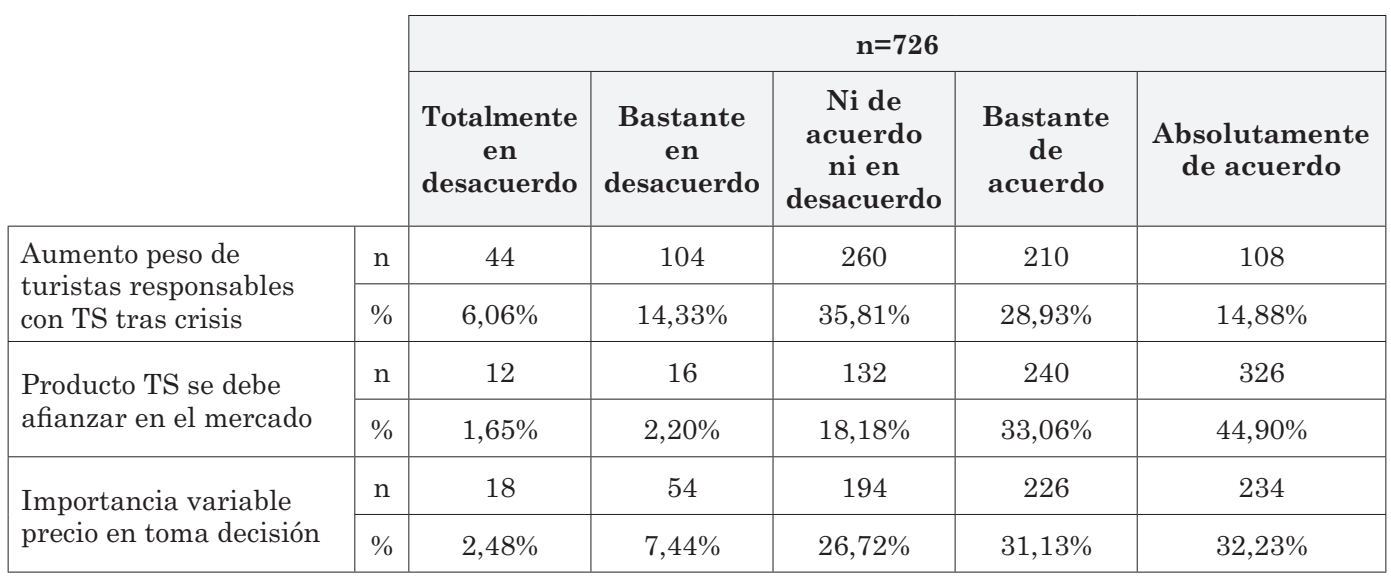

Fuente: Elaboración propia

Esta crisis podría verse como una oportunidad para reconsiderar la trayectoria de crecimiento del turismo y cuestionar la tendencia de que más llegadas de turistas implican mayores beneficios. Se antoja, por lo tanto, fundamental para el desarrollo de un tipo de turismo más sostenible, que las agencias de viajes se vayan adaptando a los estándares de calidad establecidos. Y los tour operadores, creadores de la mayoría del producto que comercializan las agencias de viajes, son los que deben adaptarse en primer lugar para poder ofertar al cliente un tipo de producto más acorde con los tiempos actuales y venideros que la etapa post COVID-19 plantea (Rivera y Pastor, 2020)

La cadena de valor de la comercialización turística obliga a todos los implicados a colaborar entre sí y que se impliquen en la distribución de un producto más sostenible. El ciclo de vida del viaje turístico debe tener en cuenta el concepto de sostenibilidad en cada una de sus etapas; el sueño, la búsqueda, la reserva, la experimentación y el intercambio de información (Pastor, 2019) estando la agencia de viajes está presente en cada una de ellas.

En el estudio realizado por Rivera y Pastor (2020) se constata que las agencias de viajes no han sabido absorber la demanda de un producto más sostenible y por este motivo el agente de viajes se ha sentido desplazado de este mercado, ya sea por desconocimiento o por la inercia de venta de determinados productos más habituales. Los autores concluyen que los operadores actuales que distribuyen el producto turístico a través de las agencias de viajes, no han sido capaces de aglutinar este tipo de producto y distribuirlo de forma apropiada para llegar a un mayor tamaño del mercado. El estudio afirma que los agentes de viajes están abiertos a recibir formación en este particular y creen del todo necesario la creación de productos sostenibles ya que consideran que el mercado actual lo demanda.

\subsection{Desintermediar lo máximo posible en beneficio propio y del cliente.}

En los últimos años, la transformación de la distribución turística ha creado un nuevo y complejo mapa multicanal en el que están surgiendo nuevos agentes y nuevas estrategias de marketing (Fernández-Villarán, A. et al., 2020).

Internet ha permitido, por un lado, la comunicación efectiva entre proveedores de servicios turísticos y clientes geográficamente distantes, reduciendo el porcentaje de personas que recurren a intermediarios para organizar sus viajes, y por otro lado el impacto de las nuevas tecnologías ha modificado el concepto de intermediación (Alberto et al., 2011). Los intermediarios tradicionales se enfrentan a una nueva realidad a la que deben adaptarse y, en muchos casos, tienen que modificar su modelo de negocio ante 
las nuevas realidades. El desarrollo de las TIC no ha reducido el número de intermediarios, sino que ha modificado la tradicional cadena de valor de la distribución turística incluyendo nuevos participantes de carácter eminentemente tecnológico y como consecuencia, se ha originado una red cada vez más compleja de intermediarios y canales de distribución paralelos (Rojas et al., 2020). La estructura de la industria del turismo se ha convertido una red global compleja. En la lucha por prosperar en este entorno, las partes interesadas en la cadena de valor continúan compitiendo, cooperando, fusionándose, formando asociaciones y cambiando las relaciones de forma regular (Jørgensen, 2017; Kracht y Wang, 2010;). A medida que el mercado va madurando, los canales de distribución se vuelven más complejos y las relaciones entre las diferentes partes interesadas tienden a desarrollarse para mejorar la eficiencia (Jørgensen, 2017).

La cadena de valor de la distribución turística se ha modificado y, por lo tanto, es lícito tanto por parte de los tour operadores como de las agencias de viajes, tratar de buscar la mayor rentabilidad posible, así como el mayor beneficio para el cliente. Por supuesto, lo ideal sería conseguir el equilibrio entre todos y que la colaboración entre empresas del sector fluya de manera natural y sea la mayor posible. Las agencias de viajes deben contar con este particular a la hora de enfrentarse a la nueva realidad. Las herramientas que los proveedores han puesto a su servicio durante mucho tiempo en exclusiva para mejorar los procesos de cotización, visualización de plazas etc. pueden ponerse de la misma manera a disposición del cliente final para fomentar un proceso de compra directa. Y del mismo modo que los agentes de viajes hace tiempo que recurren a los proveedores (hoteles, agencias receptivas o DMC, empresas de transporte, etc.) de los propios tour operadores con los que trabajan habitualmente, éstos podrán dirigirse directamente al cliente final. Dependerá del cliente optar por la compra en una agencia de viajes si ésta le ofrece un valor añadido en cuanto a filtración de la información, personalización del viaje, trato ofrecido, etc. o, por el contrario, utilizar las opciones de venta del tour operador, normalmente virtuales y sin el contacto humano que puede decantar la venta hacia uno u otro lado.

Esto es más actual si cabe, si nos referimos al producto sostenible. Las agencias que deseen, ya sea por iniciativa propia o a petición del cliente, ofrecer un tipo de producto más respetuoso con el medio ambiente deberán buscarlo o crearlo de manera individual ya que actualmente hay una falta de este producto en el mercado que pueda ser comercializado por las agencias. De hecho, como se puede observar en la tabla 3, solo un 10,2\% de los encuestados consideran lo contrario. Además, entienden que solo un $7,16 \%$ del tour operadores actuales están preparados a nivel de producto e información para afrontar este auge de turismo sostenible y un contundente $73,9 \%$ opina que, para poder atender mejor a este tipo de cliente, serían necesarios en el mercado operadores especializados que aglutinen la oferta de producto sostenible (TS) dispersa que existe en la actualidad.

Tabla 4: Percepción acerca del producto turístico sostenible

\begin{tabular}{|c|c|c|c|c|c|c|}
\hline & \multicolumn{5}{|c|}{$n=726$} \\
\hline & & $\begin{array}{l}\text { Totalmente } \\
\text { en } \\
\text { desacuerdo }\end{array}$ & $\begin{array}{c}\text { Bastante } \\
\text { en } \\
\text { desacuerdo }\end{array}$ & $\begin{array}{c}\text { Ni de } \\
\text { acuerdo } \\
\text { ni en } \\
\text { desacuerdo }\end{array}$ & $\begin{array}{l}\text { Bastante } \\
\text { de } \\
\text { acuerdo }\end{array}$ & $\begin{array}{l}\text { Absolutamente } \\
\text { de acuerdo }\end{array}$ \\
\hline \multirow{2}{*}{$\begin{array}{l}\text { Existencia producto TS } \\
\text { en mercado }\end{array}$} & $\mathrm{n}$ & 186 & 276 & 190 & 46 & 28 \\
\hline & $\%$ & $25,62 \%$ & $38,02 \%$ & $26,17 \%$ & $6,34 \%$ & $3,86 \%$ \\
\hline \multirow{2}{*}{$\begin{array}{l}\text { Suficiente información/ } \\
\text { formación para agentes } \\
\text { sobre TS en mercado }\end{array}$} & $\mathrm{n}$ & 228 & 288 & 134 & 54 & 22 \\
\hline & $\%$ & $31,40 \%$ & $39,67 \%$ & $18,46 \%$ & $7,44 \%$ & $3,03 \%$ \\
\hline \multirow{2}{*}{$\begin{array}{l}\text { Capacitación en TS } \\
\text { suficiente en operadores } \\
\text { convencionales }\end{array}$} & $\mathrm{n}$ & 196 & 324 & 154 & 34 & 18 \\
\hline & $\%$ & $27,00 \%$ & $44,63 \%$ & $21,21 \%$ & $4,68 \%$ & $2,48 \%$ \\
\hline \multirow{2}{*}{$\begin{array}{l}\text { Necesidad operador } \\
\text { especialista }\end{array}$} & $\mathrm{n}$ & 32 & 44 & 114 & 282 & 254 \\
\hline & $\%$ & $4,41 \%$ & $6,06 \%$ & $15,70 \%$ & $38,84 \%$ & $34,99 \%$ \\
\hline
\end{tabular}

Fuente: Elaboración propia 


\subsection{Amplio conocimiento de los productos. Formación continua.}

Se debe tener un perfecto conocimiento del producto y de los derivados que demanda el mercado. Para ello la formación continua es esencial. El agente de viajes actual debe estar muy por encima del conocimiento de su cliente, de lo contrario su trabajo sería irrelevante ya que no aportaría ningún valor añadido. De esta manera el cliente debe sentirse en todo momento confiado y respaldado. El desarrollo tecnológico debe estar acompañado de un profundo conocimiento del producto y las innovaciones tecnológicas pueden ayudar a ello. Con la aparición de nuevas tecnologías, el sistema y los procesos del turismo también están cambiando y el agente de viajes debe estar al día de estos cambios y aprovecharse de ellos (Happ \& Ivancsó-Horváth, 2018).

En el estudio se constata que la mayoría de los encuestados tienen estudios universitarios o de formación profesional para ejercer su profesión. Entre ellos es habitual asistencia a seminarios de producto o a talleres de manera presencial y más recientemente a webinars de manera remota para complementar su formación y el conocimiento de productos.

Tabla 5: Estudios realizados por los encuestados.

\begin{tabular}{|l|r|r|}
\hline ESO o BUP & 28 & $3,90 \%$ \\
\hline Bachillerato o COU & 84 & $11,60 \%$ \\
\hline FP & 70 & $9,60 \%$ \\
\hline Diplomatura & 318 & $43,80 \%$ \\
\hline Licenciatura o Grado & 140 & $19,30 \%$ \\
\hline Master o posgrado & 84 & $11,60 \%$ \\
\hline Doctorado & 2 & $0,30 \%$ \\
\hline
\end{tabular}

Fuente: Elaboración propia

\subsection{Trabajar de manera eficiente la información}

Una de las razones más importantes por las que el viajero acudirá a una agencia de viajes será para dejar en manos de profesionales el análisis y el filtrado de la gran cantidad de información recibida por diferentes medios. La agencia de viajes deberá depurar toda esa información en función de los gustos y necesidades del cliente. Para ello deberá conocerlos y tener bien claro cuáles son esos gustos y esas necesidades para ofrecerle el producto que más se ajuste a sus demandas. Para el exceso de información, ya en 1996, sin la utilización de internet de manera masiva, Alfons Cornellá introdujo el término infoxicación para denominar el crecimiento exponencial de información que intoxica a los ciudadanos (Casas-Mas, 2014). También se conoce como infosaturación en relación con los efectos cognitivos que produce el acceso a grandes cantidades de información que el individuo no logra apropiar (Dias, 2014).

Actualmente, la cantidad de información que el cliente puede conseguir por sí mismo, unido a las facilidades de compra por parte de diferentes actores en el proceso de comercialización y distribución turística, ponen en cuestión la utilidad de las agencias de viajes, su interés como intermediario y por consiguiente su supervivencia. La dinámica competitiva generada por el uso de internet conduce a cambios importantes en la posición estratégica de cada integrante en la cadena tradicional de distribución de la industria turística (Pestek y icic, 2010) y la agencia de viajes no queda al margen. Será labor del agente de viajes revertir esta situación ofreciendo la posibilidad de cribar todo ese exceso de información para liberar al cliente de esta labor y poder elegir su viaje de acuerdo a sus gustos o necesidades. De esta manera la utilidad de la agencia de viajes será vista por el ciudadano como una opción importante a la hora de contratar un servicio turístico a pesar de poderlo hacer a través de las múltiples opciones que se ofrecen en internet.

\subsection{Desarrollo de un modelo mixto de agencias.}

La agencia de viajes desarrollará un modelo mixto entre presencial y online donde los clientes podrán optar por una presencia más personal si así lo desean, pero sin tener que desplazarse hasta la agencia física cuando necesiten realizar alguna gestión. Las nuevas tecnologías, que también dominan muchos 
clientes, harán que las transacciones puedan realizarse vía telemática. De esta manera también se desarrolla la posibilidad de acceder a nuevos mercados aumentando el beneficio de la empresa ya que se elimina la limitación física.

La pandemia ha hecho que muchos ciudadanos hayan descubierto diferentes herramientas de conectividad. Los días que la ciudadanía ha pasado confinada, han hecho que la ciudadanía haya descubierto diferentes herramientas para estar en contacto con otras personas a través de una tableta, un ordenador o un teléfono inteligente. La facilidad y comodidad en su uso las han convertido en herramientas, que mejoradas, se mantendrán en el futuro afectando tanto a las relaciones personales como profesionales. Esto abre un abanico de posibilidades muy grandes a las agencias de viajes ya que puede conectar con cualquier posible cliente en cualquier parte del mundo. Además de poder reducir los gastos fijos que supone un local, puede amoldar los horarios a sus necesidades y las de los clientes, organizando reuniones en horarios más compatibles con las obligaciones de unos y de otros. Por ello, la posibilidad de convertir la agencia tradicional física en un modelo mixto, en el que el contacto humano, ya sea vía telemática o presencial y la venta online puedan convivir, se convierte en una de las posibilidades más interesantes y con mayor proyección que nos ha dejado la situación post pandemia. Algunos directivos y profesionales reconocidos del sector, ratifican este cambio de modelo (Hosteltur, 2020).

Por otro lado, también se modificará el modelo de negocio. En la tabla 5 encontramos los siguientes resultados con relación al diferente subsector dentro de las agencias de viajes:

\section{Tabla 6: Subsector dentro de las agencias de viajes}

\begin{tabular}{|l|l|r|r|}
\hline \multirow{5}{*}{ Subsector Agencia } & Mayorista & 204 & $28,10 \%$ \\
\cline { 2 - 4 } & Minorista vacacional & 368 & $50,70 \%$ \\
\cline { 2 - 4 } & Receptivo & 32 & $4,40 \%$ \\
\cline { 2 - 4 } & Corporativo/Empresas & 58 & $8,00 \%$ \\
\cline { 2 - 4 } & Mayorista - minorista & 28 & $3,90 \%$ \\
\cline { 2 - 4 } & Otros & 36 & $5,00 \%$ \\
\hline
\end{tabular}

Fuente: Elaboración propia

Si bien la agencia online está creciendo en los últimos años, las agencias de viajes tradicionales son físicas, es decir, relación presencial con los clientes en un punto físico determinado. En los próximos años veremos crecer otro modelo de negocio B2C (de las siglas en inglés business to consumer empresa a consumidor) como es el de los "agentes de viajes en sus casas" o Home-based travel agents (HBTA, por sus siglas en inglés). Los HBTA son un modelo de negocio que, si bien no es novedoso, en España aún no está muy desarrollado. De esta manera, asesores de viajes independientes, utilizando los principios de la economía colaborativa de plataforma, reciben una comisión por la venta de determinados productos por parte de una agencia anfitriona (Host agency) que, sin tenerlos contratados en régimen general, permiten el uso de su nombre, su licencia y se beneficia de los contratos firmados con los proveedores. De esta manera no es necesaria la presencia física en un local con el ahorro de gastos fijos que conlleva.

\subsection{Personalización de los viajes}

Se ha recurrido mucho a la especialización de las agencias de viajes, pero es más importante la personalización de los mismos. Para ello es fundamental el conocimiento de los clientes como ya se ha dicho anteriormente. Ofrecer productos novedosos e inimaginables que incrementen el valor añadido de la agencia de viajes a la hora de gestionar los viajes de sus clientes. Actuar en modo "concierge" durante su viaje haciéndoles sentir únicos, respaldados y en todo momento atendidos ante los posibles problemas que surjan, se antoja como algo fundamental para conseguir la fidelización del cliente.

Las nuevas tecnologías también pueden ayudar a la realización de este particular. Una especialización puede ser interesante, pero limita mucho el acceso a diferentes nichos de mercado. La personalización por el contrario se debe ofrecer a cualquier persona que se interese por la empresa en cualquier momento. Esta personalización debe estar presenta en todas las fases del viaje. Si estructuramos el viaje en un ,antes', ,durante' y ,después', el ,antes' es la clave para iniciar la relación con el cliente, ya que es en 
esta etapa que el consumidor turístico se inspira en el destino, realiza consultas, decide y compra. Esto es seguido de la etapa „durante“ del viaje que es la propia realización del viaje. En la etapa final (,después“), las redes sociales juegan un papel predominante. Independientemente del tipo de turista, todo aquel que va a viajar pasa por las mismas etapas, emociones y pensamientos (Fernández-Villarán et al., 2020). De esta manera y basándonos en los modelos más actuales que tienen en consideración el factor tecnológico y la importancia de internet en el proceso del ciclo de vida del viaje, podemos diferenciar seis etapas diferentes (Pastor y Rivera, 2021):

$$
\begin{aligned}
& \text { - Soñar } \\
& \text { - Planificar } \\
& \text { - Comparar } \\
& \text { - Reservar } \\
& \text { - Experimentar } \\
& \text { - Compartir }
\end{aligned}
$$

En todas y cada una de ellas el concepto de personalización es importante. Hacer soñar al cliente mediante acciones en redes sociales o cualquier otra forma de marketing; ayudar a planificar el viaje adaptándolo a sus necesidades; comparar las diferentes alternativas; reservar el viaje y ofrecer los métodos de pago más cómodos o que mejor se adapten a las necesidades del cliente; estar presente durante la experimentación del viaje y tratar de conseguir que en el momento de compartir la experiencia, el trabajo del asesor sea reconocido así hacer soñar a futuros clientes potenciales.

\subsection{Ampliar los nichos de mercado; especialmente al público joven.}

En los últimos años y ante el desarrollo de las nuevas tecnologías, el público joven ha dejado de acudir a las agencias de viajes y éstas han terminado olvidándose de este grupo centrándose sobre todo en el cliente de mediana y de mayor edad que, quizás, ven en las nuevas tecnologías una barrera que ha llegado demasiado tarde para ellos requiriendo una atención más presencial. Habría que actuar y atraer a ese público joven con las mismas herramientas que utiliza habitualmente este segmento de la sociedad, cuyos hábitos de consumo y los problemas asociados no deben ignorarse y por lo tanto estar al día con los desarrollos tecnológicos para conocer cómo son utilizadas por este potencial público. Es un segmento meramente visual, para los que las fotos y sobre todo el vídeo es imprescindible. De ahí la importancia de los desarrollos de imagen de realidad virtual (Şchiopu et al., 2016) que se han citado anteriormente. Comparten en redes sociales muchas imágenes que influyen en otras personas a la hora de elegir o soñar con un destino dando comienzo al ciclo del viaje.

Las nuevas generaciones tienen nuevas necesidades sociales que satisfacer. Las vacaciones ya no se consideran únicamente como un escape de la rutina diaria, sino que se los considera cada vez más como un instrumento asociado a la cultura, el descubrimiento y la construcción de identidad. Esta idea se puede atribuir a la cantidad de información disponible en la red que alimenta la necesidad de ampliar los horizontes y la experiencia de los viajeros. Este segmento muestra una tendencia contraria al turismo masivo, diferenciándolo de un turismo más personalizado y más basado en experiencias. Las nuevas generaciones ven los viajes como herramientas capaces de enriquecerlos, de impactar en sus vidas y en sus identidades. Los jóvenes turistas sienten curiosidad por entrar en contacto con otras realidades y conocer diferentes culturas y están cada vez más acostumbrados a organizar sus vacaciones de forma independiente y siendo muy exigentes y críticos con respecto a la oferta turística actual (Monaco, 2018).

Por otro lado, es el segmento más concienciado con la sostenibilidad, por lo que el desarrollo de nuevos productos puede venir de su mano (Trobat, et al., 2005). Según Rainer (2011) 9 de cada 10 nacidos entre 1980 y 2000 dicen sentirse responsables con el mundo en el que vivimos y son conscientes de que deben de hace un cambio del paradigma actual. De la misma manera, buscan disminuir el impacto ejercido sobre la tierra, reducir la huella de carbono y eliminar daños innecesarios sobre el medioambiente, con vistas a garantizar el futuro de las futuras generaciones, a diferencia de las generaciones anteriores que se han movido más por motivaciones cortoplacistas y meramente económicas (Pendergast, 2010).

\subsection{Omnicanalidad}

Con la proliferación actual de diferentes opciones de canales, la industria de los viajes debe esforzarse en atraer a los clientes en todos ellos. Los sistemas de servicios multicanal se han vuelto cada vez más importantes con el auge del comercio electrónico (Patten, 2017). De hecho, la venta omnicanal es un medio muy importante para forjar las relaciones con los clientes y para el desarrollo de nuevos mercados 
(Mika et al., 2018). El viajero ya nunca será sólo presencial ya que su dinámica diaria es omnicanal. Por ello la agencia debe dejar de dividirse entre offline y online y derivar a un modelo mixto y utilizar todos los canales que tenga a su alcance para conseguir una propuesta de valor a todos los públicos.

El proceso de transformación de la distribución turística ha dado lugar a un nuevo mapa multicanal, complejo, en el que surgen nuevos actores y se desarrollan nuevas estrategias de marketing, pero que es necesario comprender para poder tomar decisiones de comercialización de forma adecuada (Rodríguez-Zulaica, et al., 2017). Existe la necesidad de ofrecer diferentes opciones de compra a través de múltiples canales y dispositivos con la finalidad de atender las necesidades del cliente multicanal. (Vallespín, et al., 2017).

En el estudio realizado por Rivera y Pastor (2020) se indica que el uso por parte de las agencias de viajes de redes sociales y herramientas de mensajería instantánea es de un $92 \%$. En esta línea el estudio de Pastor (2019) sobre su utilización profesional indica que casi el 90\% consideran que son una buena herramienta de venta, aunque casi la mitad lo ven sólo como un medio publicitario y no como una herramienta que facilite la transacción comercial.

Otro estudio corrobora estos datos e indica que, de media, las agencias utilizan tres medios diferentes para su relación con sus clientes. La relación personal, el correo electrónico y el teléfono son los tres más habituales, siendo la aplicación WhtasApp la más utilizada después de la relación personal, el correo electrónico y el teléfono. Sólo un 16\% utiliza los medios y las redes sociales para relacionarse con los clientes (Observatur, 2019).

Actualmente el consumidor puede considerarse multicanal ya que durante su proceso de compra utiliza diferentes medios y/o canales. Desde el momento en el que se despierta el interés por un destino a través de cualquier medio, su primera acción es la de ampliar información en una web, para después planificar y comparar alternativas en una agencia de viajes o en las múltiples opciones que ofrece la red, reservarlo y pagarlo desde el reloj inteligente o por medios de pago electrónicos y compartir su experiencia en las redes sociales a través de su teléfono inteligente, estando en contacto con su asesor de viajes mediante herramientas o aplicaciones de mensajería instantánea. En todas las fases del ciclo del viaje se puede observar cómo son utilizados todo tipo de canales.

\section{Conclusiones, limitaciones y futuras líneas de investigación}

Durante la pandemia, las agencias de viajes, especialmente las que se dedican al segmento vacacional, así como los tour operadores, han permanecido en una especie de letargo ante la imposibilidad de poder atender a los clientes ya que los confinamientos, cierres de fronteras, cancelación de vuelos y cierre de buena parte de la hotelería han privado a éstas de clientes a los que poder atender. Muchas han optado por cerrar temporalmente, otras definitivamente y otras han permanecido abiertas durante determinadas horas al día. En todos los casos, han sido uno de los sectores que más han sufrido las consecuencias de la crisis producida por la pandemia. Según todos los datos, la vuelta a la pasada normalidad aún tardará y en todo caso no va a ser la misma. Las agencias de viajes deben de evolucionar como ya lo hicieron tras la irrupción de internet en nuestras vidas, quizás el cambio más importante que han sufrido hasta el momento. Ni los clientes serán iguales, ni las relaciones con ellos serán las mismas. La pandemia ha obligado a avanzar en la digitalización de la sociedad de una manera más rápida de lo esperada y las agencias tienen que ser conscientes de ello una vez la reapertura sea general. Por otro lado, el turismo, será sostenible o no será por lo que se debe avanzar en la creación, distribución y venta de este producto, por el bien de todos y porque el cliente así lo exigirá ya que en muchas ocasiones se relacionará con el free-Covid. Aparecerán nuevos modelos de negocio y nuevos modelos mixtos de agencias que den servicio de manera presencial y virtual. Se tenderá a la desintermediación, lo que no significará la desaparición de las agencias de viajes, sino que convivirán diferentes formas de relación con el cliente. En todo caso la omnicanalidad o multicanalidad será un hecho ante el auge de las TIC y el conocimiento de muchas herramientas que la sociedad ha adquirido de manera muy rápida e inimaginable poco tiempo atrás durante la pandemia. Estas herramientas, además, pueden ayudar a la formación continua del agente y de esta manera poder personalizar de manera más eficiente el viaje. El cliente es y será el centro de toda actuación y se debe evolucionar con él. Las agencias de viajes tienen futuro, pero ese futuro dependerá de lo que cada una de ellas pueda adaptarse a las nuevas demandas del mercado.

Una de las limitaciones más importantes que se ha experimentado ha sido la dificultad de establecer contacto con un mayor número de agentes de viajes, por cuanto la pandemia ha golpeado duramente a este sector y miles de ellos se encuentran en situaciones laborales que les alejan de sus puestos de trabajo habituales, desde expedientes de regulación de empleo temporal (ERTE) o despidos (ERE), 
hasta cierres temporales o definitivos de sus agencias. En cualquier caso, agradecemos la voluntad de todos los que han hecho posible este trabajo.

Emplazamos a futuras investigaciones la confirmación de la validez de esta propuesta con datos objetivos, así como el estudio de los modelos de negocio nuevos o menos desarrollados en nuestro mercado que pueden emerger tras la pandemia, entre los que se encuentran los "agentes de viajes en sus casas" o HBTA. Los HBTA son uno de los modelos de distribución de viajes menos definidos e investigados tanto entre los profesionales de los viajes como entre los académicos (Bowden, 2008). Un modelo que, sin ser disruptivo, si es de escasa implantación en España, pero al que se le prevé un incremento por su adecuación a los tiempos de crisis como se ha podido constatar en otros mercados como USA o Canadá.

\section{Referencias}

Alberto, D. F., Niebla, L. S., y Turégano, M. Á. S. 2011. ¿Desaparecerán los Tour Operadores? El papel de los intermediarios en la distribución turística: Análisis del caso de Tenerife. PASOS Revista de Turismo y Patrimonio Cultural, 9(2), 341-351.

Almeida, M. D. M. A. 2019. Robots, inteligencia artificial y realidad virtual: una aproximación en el sector del turismo. Cuadernos de Turismo, (44), 13-26.

Bakar, N. A., \& Rosbi, S. 2020. Effect of Coronavirus disease (COVID-19) to tourism industry. International Journal of Advanced Engineering Research and Science, 7(4), 189-193.

Bowen, J., \& Whalen, E. 2017. Trends that are changing travel and tourism. Worldwide Hospitality and Tourism Themes.

Buhalis, D. 2000. Tourism and information technologies: Past, present and future. Tourism recreation research, 25(1), 41-58.

Buhalis, D., \& Licata, M. C. 2002. The future eTourism intermediaries. Tourism management, 23(3), 207-220.

Casas-Mas, B. 2014. Infoxicación a través de los medios de comunicación. Ámbitos: Revista Internacional de Comunicación, 24, 1-11.

Cheer, J. M. 2020. Human flourishing, tourism transformation and COVID-19: A conceptual touchstone. Tourism Geographies, 22(3), 514-524.

Coyle, D., \& Yeung, T. 2016. Understanding AirBnB in fourteen European cities. The Jean-Jacques Laffont Digital Chair Working Papers, 7088, 1-33.

Dias, P. 2014. From 'infoxication'to 'infosaturation': a theoretical overview of the cognitive and social effects of digital immersion. Ambitos, 24.

Doherty, N. F., Ellis-Chadwick, F., \& Hart, C. A. 1999. Cyber retailing in the UK: the potential of the Internet as a retail channel. International Journal of Retail \& Distribution Management.

Fernández-Villarán, A., Rodríguez-Zulaica, A., \& Pastor, R. 2020. Value chain mapping for tourism intermediation. International Journal of Business Environment, 11(2), 81-97.

Gomis, J. M., \& de Borja, L. 2006. Modelos de innomediación en el marco de un nuevo paradigma de la intermediación turística. VI CongresoNacional "Turismo y Tecnologías de la Información y lasComunicaciones, 213-226.

Gössling, S., Scott, D., \& Hall, C. M. 2020. Pandemics, tourism and global change: a rapid assessment of COVID-19. Journal of Sustainable Tourism, 29(1), 1-20.

Hall, C. M. 2019. Constructing sustainable tourism development: The 2030 agenda and the managerial ecology of sustainable tourism. Journal of Sustainable Tourism, 27(7), 1044-1060.

Happ, E., \& Ivancsó-Horváth, Z. 2018. Digital tourism is the challenge of future-a new approach to tourism. Knowledge Horizons. Economics, 10(2), 9-16.

Hosteltur. Las agencias de viajes mutarán a un modelo mixto tras el coronavirus. 2020, junio. Recuperado de https://www.hosteltur.com/137143_las-agencias-de-viajes-mutaran-a-un-modelo-mixto-tras-el-coronavirus.html

Huang, C., Wang, Y., Li, X., Ren, L., Zhao, J., Hu, Y., ... \& Cao, B. 2020. Clinical features of patients infected with 2019 novel coronavirus in Wuhan, China. The lancet, 395(10223), 497-506.

Jiang, Y., \& Wen, J. 2020. Effects of COVID-19 on hotel marketing and management: a perspective article. International Journal of Contemporary Hospitality Management.

Jones, L. D., Golan, D., Hanna, S. A., \& Ramachandran, M. 2018. Artificial intelligence, machine learning and the evolution of healthcare: A bright future or cause for concern? Bone \& Joint Research, 7(3), $223-225$. 
Jørgensen, M. T. 2017. Reframing tourism distribution-activity theory and actor-network theory. Tourism Management, 62, 312-321.

Kracht, J., \& Wang, Y. 2010. Examining the tourism distribution channel: evolution and transformation. International Journal of Contemporary Hospitality Management.

Kunzmann, K. R. 2020. Smart Cities After Covid-19: Ten Narratives. disP-The Planning Review, 56(2), 20-31.

Mallar, M. Á. 2010. La gestión por procesos: un enfoque de gestión eficiente. Revista Científica" Visión de Futuro", 13(1).

Molina, C. 2020, 12 octubre. Las agencias de viajes se alian para exigir en los tribunales 500 millones a las aerolíneas. Cinco Días. Recuperado de: https://cincodias.elpais.com/cincodias/2020/10/09/ companias/1602231080_193379.html\#:\%7E:text=Una\%20ca\%C3\%ADda\%20de\%20la\%20 facturaci\%C3\%B3n\%20 del\%2090\%25\%20en\%202020\&text=Las\%20previsiones\%20de\%20 facturaci $\%$ C3\%B3n $\% 20$ de,de $\% 20$ hasta $\% 20 \mathrm{el} \% 2090 \% 25 \% \mathrm{E} 2 \% 80 \% 9 \mathrm{D}$

Monaco, S. 2018. Tourism and the new generations: emerging trends and social implications in Italy. Journal of Tourism Futures.

Observatur. (Observatorio Nacional del turismo emisor). [en línea] 2019. El papel de las agencias de viajes del futuro. Recuperado de http://www.observatur.es/observatur/\#descarga

Parrales, E. C. R., \& Zambrano, L. I. B. 2017. Posicionamiento SEO mediante la optimización de sitios web para el marketing digital. Pro Sciences: Revista de Producción, Ciencias e Investigación, 1(1), 6-9.

Pastor Ruiz, R. 2019. Las agencias de viajes tradicionales en España frente al reto de la nueva intermediación. Nuevas formas de relación con clientes y proveedores. (Doctoral dissertation, Universidad Rey Juan Carlos).

Pastor Ruiz, R. y Rivera García, J. 2022. Stages of travel, in Buhalis, D. (ed.), Encyclopaedia of Tourism Management and Marketing. Edward Elgar Publishing, Cheltenham. (in press).

Pastor, R., \& Rivera-García, J. 2020. Airbnb y la intermediación turística. ¿Competición o coopetición? Percepción de los agentes de viajes en España. Revista Empresa y Humanismo, 107-132.

Patten, E. 2017. Conceptualizing service quality in multichannel fashion retailing (Doctoral dissertation, University of Gloucestershire).

Pendergast, D. 2010. Getting to know the Y generation. Tourism and generation Y, 1, 1-15.

Peštek, A., \& Cicic, M. 2010. Application of e-marketing strategies in tourism sector in Bosnia and Herzegovina. In Proceedings, 5th International Conference "An Enterprise Odyssey: From Crisis to Prosperity-Challenges for Government and Business (pp. 132-145).

Rainer, T. S., \& Rainer, J. 2011. The millennials. B\&H Publishing Group.

Rivera García, J. \& Ruíz, R. P. 2020. ¿Hacia un turismo más sostenible tras el Covid-19? Percepción de las agencias de viajes españolas. Gran tour, revista de investigaciones turísticas, (21).

Rodríguez-Zulaica, A., Pastor, R., \& Ara, M. A. F. V. 2017. Evolución de la intermediación turística en España tras la aparición de las TIC en el sector. Revista empresa y humanismo, 87-106.

Rojas Bueno, A., Alarcón Urbistondo, P., \& del Alcázar Martínez, B. 2020, May. The MICE tourism value chain: Proposal of a conceptual framework and analysis of disintermediation. In Journal of Convention \& Event Tourism (Vol. 21, No. 3, pp. 177-200). Routledge.

Şchiopu, A. F., Pădurean, A. M., Ţală, M. L., \& Nica, A. M. (2016). The influence of new technologies on tourism consumption behavior of the millennials. Amfiteatru Economic Journal, 18(Special Issue No. 10), 829-846.

Trobat, M. F. O., Castells, M., Casero, A., \& Morey, M. (2005). Actitudes y percepción del medio ambiente en la juventud española. Organismo Autónomo Parques Nacionales.

Vallespín, M., Molinillo, S., \& Aranda, J. P. (2017). Análisis de los hábitos de la generación Y en la planificación de viajes en un contexto multicanal. Revista de Análisis Turístico, (23), 52-60.

World Health Organization. (2020). Considerations for implementing and adjusting public health and social measures in the context of COVID-19: interim guidance, 4 November 2020 (No. WHO/2019-nCoV/ Adjusting_PH_measures/2020.2). World Health Organization.

Yrjölä, M., Spence, M. T., y Saarijärvi, H. (2018). Omni-channel retailing: propositions, examples and solutions. The International Review of Retail, Distribution and Consumer Research, 28(3), 259-276.

Recibido:

$12 / 01 / 2021$

Reenviado:

$02 / 03 / 2021$

Aceptado:

$17 / 05 / 2021$

Sometido a evaluación por pares anónimos 